\title{
Circulating Tumor DNA Analysis in Colorectal Cancer: From Dream to Reality
}

\author{
Carlotta Antoniotti, MD ${ }^{1,2}$; Filippo Pietrantonio, MD ${ }^{3,4}$; Salvatore Corallo, MD ${ }^{3}$; Filippo De Braud, MD ${ }^{3,4}$; Alfredo Falcone, MD ${ }^{1,2}$; and \\ Chiara Cremolini, MD, $\mathrm{PhD}^{1,2}$
}

Liquid biopsy is a minimally invasive approach to obtain circulating materials that originate from tumor cells through the sampling of body fluids, mainly peripheral blood. Because of its abilities to detect tumor-derived nucleic acids and proteins and to characterize tumor-specific genomic abnormalities, liquid biopsy has emerged as an approach to orient care of patients with colorectal cancer (CRC). The most advanced approach through which liquid biopsy could be exploited in the CRC field is the analysis of circulating cell-free tumor DNA (ctDNA).

ctDNA is detected in almost all patients with $\mathrm{CRC}{ }^{1}$ Available approaches to ctDNA analysis range from the interrogation of a single or a limited number of loci to whole-genome analyses. ${ }^{2}$

Several applications of ctDNA analysis have been hypothesized in the clinical scenario of CRC, including the following: molecular profiling for treatment selection, prognosis assessment and detection of minimal residual disease, monitoring of treatment efficacy and emergence of secondary resistance to ongoing therapies, and identification of candidates for antiepidermal growth factor receptor (EGFR) rechallenge. However, the role of liquid biopsy as a tool to build a biology-driven care of patients with CRC needs validation to enter clinical guidelines and recommendations. ${ }^{3,4}$

We reviewed available literature about the applications of ctDNA analysis with the aim of disclosing the main gaps that must be filled to push the development of liquid biopsy toward clinical practice.

ASSOCIATED CONTENT

Appendix

Author affiliations

and support

information (if

applicable) appear at

the end of this

article.

Accepted on January

23, 2019 and

published at

ascopubs.org/journal/

po on April 18, 2019:

DOI https://doi.org/10.

1200/PO.18.00397

\section{ctDNA Versus Tumor DNA for RAS Profiling}

Expectations are high about the possibility of using blood instead of tissue samples to detect RAS mutations for anti-EGFR (cetuximab and panitumumab) treatment selection in metastatic CRC ( $\mathrm{mCRC}$ ).

Many retrospective series have described a more than 90\% agreement between RAS status in matched tumor and ctDNA samples and have highlighted that RAS mutations are detectable in ctDNA with high specificity (90\% to 100\%), but suboptimal sensitivity (89\% to 96\%; Appendix Table A1). ${ }^{5-9}$ Nevertheless, when RAS testing on ctDNA was compared with highly sensitive tissue-based techniques, ${ }^{10,11}$ concordance rate $(78 \%$ to $88 \%)$, specificity ( $83 \%$ to $91 \%$ ), and sensitivity (70\% to $85 \%$ ) of plasma-based analyses were less encouraging (Appendix Table A1).

Retrospective data suggested that RAS testing on ctDNA results in a similar clinical outcome compared with tissue testing in patients with $\mathrm{mCRC}$ who were treated with anti-EGFR-based regimens. ${ }^{5,10,11}$ However, some issues hamper RAS genotyping on ctDNA as an alternative to tissue analysis for anti-EGFR treatment selection.

First, the standardization of preanalytic variables for ctDNA analysis is still lacking, and this may affect ctDNA quality. Second, the reliability of studies that evaluate the concordance of RAS testing between tissue and plasma samples is impaired by the adoption of assays with heterogeneous analytical sensitivity and coverage of genomic regions. There are now several commercially available methods for ctDNA assessment and technology platforms based on digital polymerase chain reaction or next-generation sequencing approaches-each one with specific sensitivity, specificity, throughput, gene coverage, costs, and potential clinical applications. ${ }^{12}$ Among them, three test kits are CE-marked in vitro diagnostic devices for detection of RAS and BRAF mutations on CtDNA in CRC. ${ }^{13-16}$

Third, some clinicopathologic variables are likely to affect the amount of tumor-released ctDNA. 5,6,8,10,11 Whereas liver involvement and tumor burden are positively associated with the RAS mutant allele fraction (the proportion of mutant DNA fragments at a given locus), peritoneal, nodal, and lung metastases and mucinous histology are linked to low RAS ctDNA detection. Factors that influence ctDNA levels, and the variability in the sensitivity of current technologies, should be considered during interpretation of RAS ctDNA results. Although lack of RAS mutations detected in ctDNA does not perfectly predict RAS wild-type status, the detection of RAS mutations is highly reliable for RAS-mutant status. Consequently, on the basis of the reported suboptimal sensitivity of ctDNA testing (around 90\%), the risk of treating with a null, if not detrimental, effect (ie, with an anti-EGFR-based therapy) false 
RAS wild-type cases, as assessed on ctDNA, is not negligible.

The parallel assessment of the mutant allele fractions of other key genomic tumor alterations may help solve challenging cases that have undetectable RAS mutations and ctDNA levels at or lower than the analytic sensitivity of adopted assays. To overcome these issues, preanalytic procedures should be standardized, a threshold of detectable mutation rate that confers intrinsic resistance to EGFR inhibition should be set and prospectively validated, and additional investigation to understand when plasma and tissue tests are interchangeable and to improve assay sensitivities is warranted.

Meanwhile, the RAS status assessment to address the use of anti-EGFR agents must be performed as the gold standard on tumor specimens. . $^{3,4,17,18}$ Only when tissuebased testing is technically or logistically unfeasible could it be replaced by ctDNA analysis. ${ }^{4}$

\section{ctDNA to Estimate Prognosis and Detect Minimal Residual Disease}

Retrospective studies have focused on the prognostic impact of the quantitative analysis of ctDNA. In the metastatic setting, a correlation between ctDNA concentration and survival has been described, but the independent weight of ctDNA quantification when the relative impact of other well-known prognostic factors is taken into account has not been clarified (Appendix Table A2).

The role of the quantitative assessment of ctDNA in monitoring response during treatment must be defined in the light of its potential added value when compared with easily available and well-established markers, including carcinoembryonic antigen or early radiologic disease reassessment. Recently, ctDNA has been proposed to detect, measure, and monitor residual disease after radical interventions.

A series of proof-of-concept studies reported that liquid biopsy could disclose the persistence of minimal residual disease (MRD) through the detection of ctDNA in patients with CRC who underwent potentially curative surgery (resection of primary tumor in early-stage CRC or radical resection of metastases) even in the absence of clinical or radiologic signs of residual disease (Appendix Table A2). By identifying incomplete eradication of disease after a curative treatment, detectable ctDNA predicts an increased risk of relapse regardless of the exposure to an adjuvant treatment. The development of such a sensitive tool might improve the risk estimation of disease relapse after a curative intervention to properly stratify clinical trials in early-stage CRC and to accordingly drive the therapeutic management.

Theoretically, two applications of the detection of MRD in this setting may be foreseen: to offer chemotherapy to all postoperative ctDNA-positive patients, including those with no histopathologic risk factors to reduce their risk of progression-indeed, ctDNA positivity invariably means residual disease — and to avoid useless adjuvant therapies in postoperative ctDNA-negative patients. Not detecting ctDNA in the postoperative does not invariably mean lack of residual disease, because available assays may have suboptimal sensitivity. As a consequence, postoperative ctDNA cannot yet be regarded as a tool to avoid the recommended treatment in ctDNA-negative patients who are candidates for adjuvant therapy according to clinical practice (ie, those with stage III and high-risk stage II CRC). ${ }^{19,20}$ The integration of different sequencing approaches, such as the detection of mutated and methylated ctDNA, could increase the sensitivity of ctDNA detection assays.

Similarly, improvement in the prognostic accuracy for those patients who undergo a radical resection of metastases is clearly needed to establish the best postoperative strategy on an individual basis. If ctDNA is detected immediately after resection, a more intensive approach may be justified.

These applications of ctDNA testing are limited by some open challenges. In early-stage CRC, ctDNA is detected at a lower rate than in the advanced disease. ${ }^{1}$ Therefore, highly sensitive techniques are needed to achieve appropriate accuracy to detect MRD.

Most available data have been achieved through a twostep procedure: the identification of specific somatic abnormalities in tissue samples, followed by the search for the same alteration in ctDNA. Currently, a panel including the most frequently and directly detected tumorspecific genomic events in ctDNA has not been validated. Only trials that aim to optimize the treatment of all postoperative ctDNA-positive patients, independent of traditional histopathologic factors, are ethically acceptable.

\section{ctDNA to Track Tumor Response and Resistance to Therapy}

Whereas tissue biopsies catch single snapshots of the tumor in a specific spatiotemporal fragment, liquid biopsy may more comprehensively depict the intrinsic and dynamic intratumoral heterogeneity. Serial quantitative and qualitative ctDNA measurements allow longitudinal exploration and tracking of the clonal evolution during and across subsequent systemic treatments. ${ }^{21-27}$

This concept especially fits with the optimization of the use of anti-EGFR agents. Indeed, patients with molecularly selected $m C R C$ who initially benefit from anti-EGFR agents almost invariably experience disease progression.

The molecular landscape of secondary resistance is heterogeneous and partially overlaps with that of primary resistance. ${ }^{28}$ Both in vitro and retrospective clinical studies have described the emergence of multiple genomic alterations both inside and outside the EGFR pathway, 
including RAS, BRAF, and EGFR ectodomain mutations and KRAS, HER2, and MET amplifications. ${ }^{29-33}$

Several researchers have used liquid biopsies to monitor treatment effect in patients who receive anti-EGFR-based therapies: the ctDNA analysis of samples collected during treatment has demonstrated the progressive selection of genomic alterations (mainly RAS mutant clones) as drivers of secondary resistance to the EGFR blockade even earlier than the radiologic evidence of disease progression. The heterogeneity and dynamism of the tumor clonal evolution under the pressure of targeted treatments are confirmed by the emergence of multiple alterations in the mitogenactivated protein kinase pathway effectors, including $R A S$ and MEK mutations and KRAS, BRAF ${ }^{\mathrm{V} 600 \mathrm{E}}$, and MET amplification, during the treatment with BRAF/EGFR, BRAF/MEK and BRAF/EGFR/MEK inhibitors in patients with $B R A F^{\mathrm{V} 600 \mathrm{E}}$-mutant $\mathrm{mCRC}$. $^{23-27}$ Two novel NTRK1 mutations have been detected as a potential mechanism of acquired resistance to entrectinib, a tyrosine kinase receptor inhibitor, in a patient with LMNA-NTRK1-rearranged mCRC. ${ }^{34}$ Nevertheless, some steps should be covered to translate liquid biopsy from the investigational setting into clinical practice.

Available data indicate that the frequency of molecular alterations in ctDNA at the time of disease progression is inconsistent among different series, even when the same methods for plasma ctDNA analysis is applied (Table 1). This inconsistency impairs the reliability of adopted techniques and the reproducibility of the findings. Setting and validation of a quantitative threshold to define the clinical relevance of each detectable molecular alteration as clearly associated with a lack of benefit from ongoing therapies may be relevant to biologically guide therapeutic decisions. Currently, no prospective data are available about the usefulness of discontinuing ongoing therapy and initiation of a tailored treatment when signals of acquired resistance emerge in ctDNA before clinical or imaging-based disease progression is noted.

The co-occurrence of multiple and/or subclonal molecular alterations at the time of acquired resistance highlights an increase in tumor heterogeneity, which complicates the definition of clinical value of each identified alteration as therapeutic target for subsequent tailored strategies. In other words, how to therapeutically target the heterogeneous mechanisms of resistance and the subclonal patterns of tumor cell populations that emerge upon drug selection is today still challenging. Most of emerging alterations are not therapeutically actionable, and the most promising approaches (eg, targeting EGFR ectodomain mutations by second-generation anti-EGFRs) have failed to prove clinical efficacy. ${ }^{47}$

\section{ctDNA to Select Patients Eligible for Anti-EGFR Rechallenge}

In patients who experienced acquired resistance to antiEGFR agents after an initial clinical benefit, and who are subsequently exposed to at least one other intervening therapy, the reintroduction of an anti-EGFR in later lines has shown promising activity data in a retrospective series. ${ }^{48}$ Recent reports disclosed a biologic rationale that supports the reintroduction of the EGFR blockade after an antiEGFR-free interval. RAS-mutated clones emerge at the time of disease progression and then decline with time upon the withdrawal of the anti-EGFR pressure, 7,38,49 which suggests a potential reversibility of the resistant phenotype.

The phase II CRICKET (Phase II Study of Cetuximab Rechallenge in Irinotecan-Pretreated $\mathrm{mCRC}, K R A S, N R A S$ and BRAF Wild-Type Treated in First Line With Anti-EGFR Therapy) trial demonstrated the activity of a cetuximabbased rechallenge strategy in patients with RAS/BRAF wild-type $\mathrm{mCRC}$ whose disease had acquired resistance to first-line cetuximab-containing therapy. ${ }^{50}$ This study highlighted the role of liquid biopsy in the selection of optimal candidates for this strategy, because the detection of RAS mutations in ctDNA collected before rechallenge is associated with no clinical benefit.

An attempt to strengthen these findings is challenged by the ongoing biomarker-driven CHRONOS (Phase II Trial of Rechallenge With Panitumumab Driven by RAS ClonalMediated Dynamic of Resistance) study, ${ }^{51}$ which adopted ctDNA analysis as an inclusion criterion. In this proof-ofconcept study, patients who are candidates for anti-EGFR rechallenge are eligible only if a notable decrease in $R A S$ fractional mutational abundance occurs from the time of disease progression after a first-line anti-EGFR-containing therapy to the time of rechallenge.

Liquid biopsy has emerged as a minimally invasive tool to genotype tumors, to assess patient prognosis and detect MRD, to monitor treatment efficacy, and to track the dynamism of clonal evolution over time and therapies. The assessment of ctDNA stands as an intriguing technology to build a personalized and biology-driven continuum of care for patients with CRC.

Awaited results of properly designed ongoing prospective clinical trials, conceived with the purpose of integrating the ctDNA analysis in the therapeutic plan for patients with CRC, could provide acceptable evidence to push liquid biopsy toward clinical practice (Appendix Table A3). 


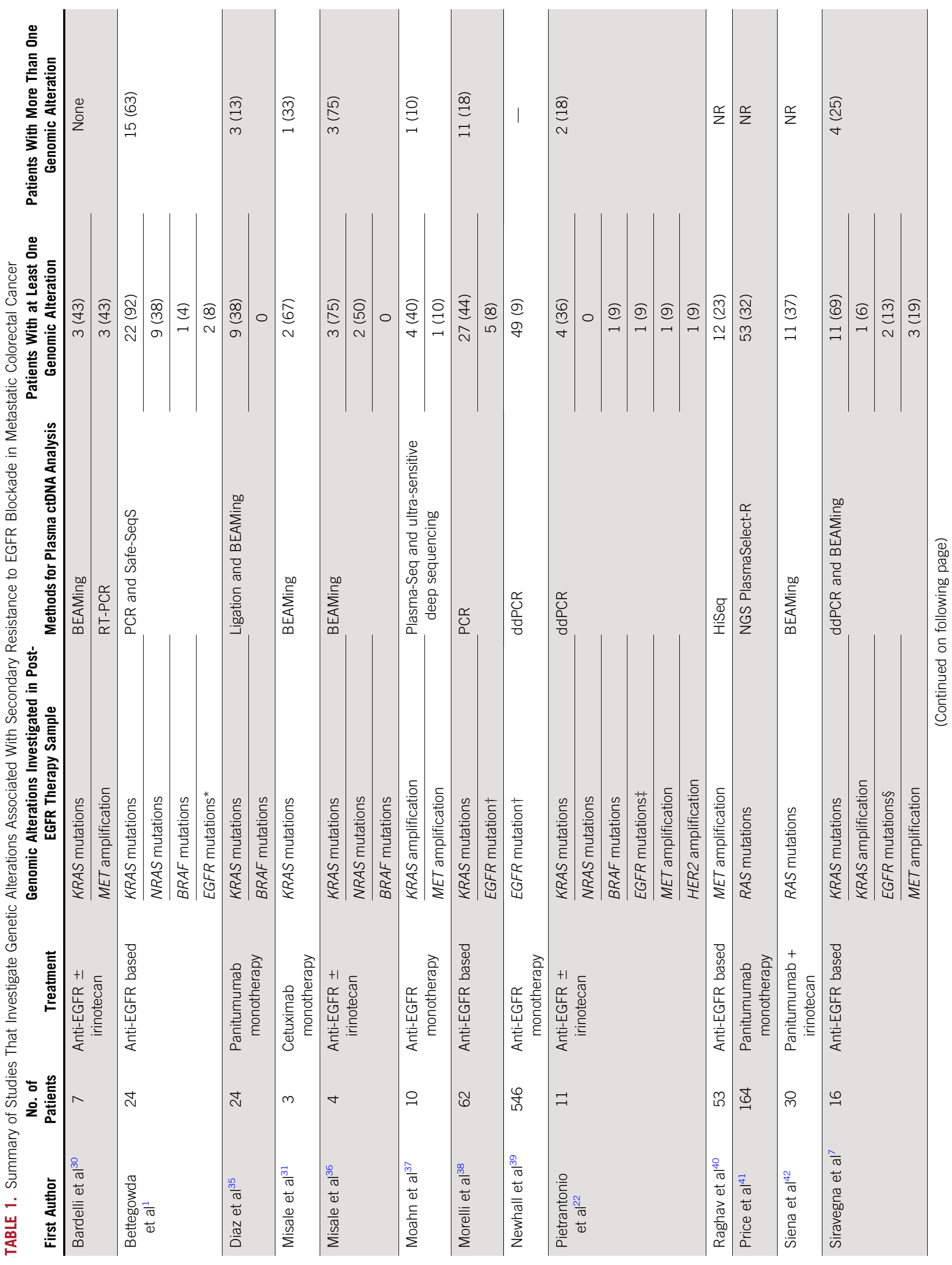




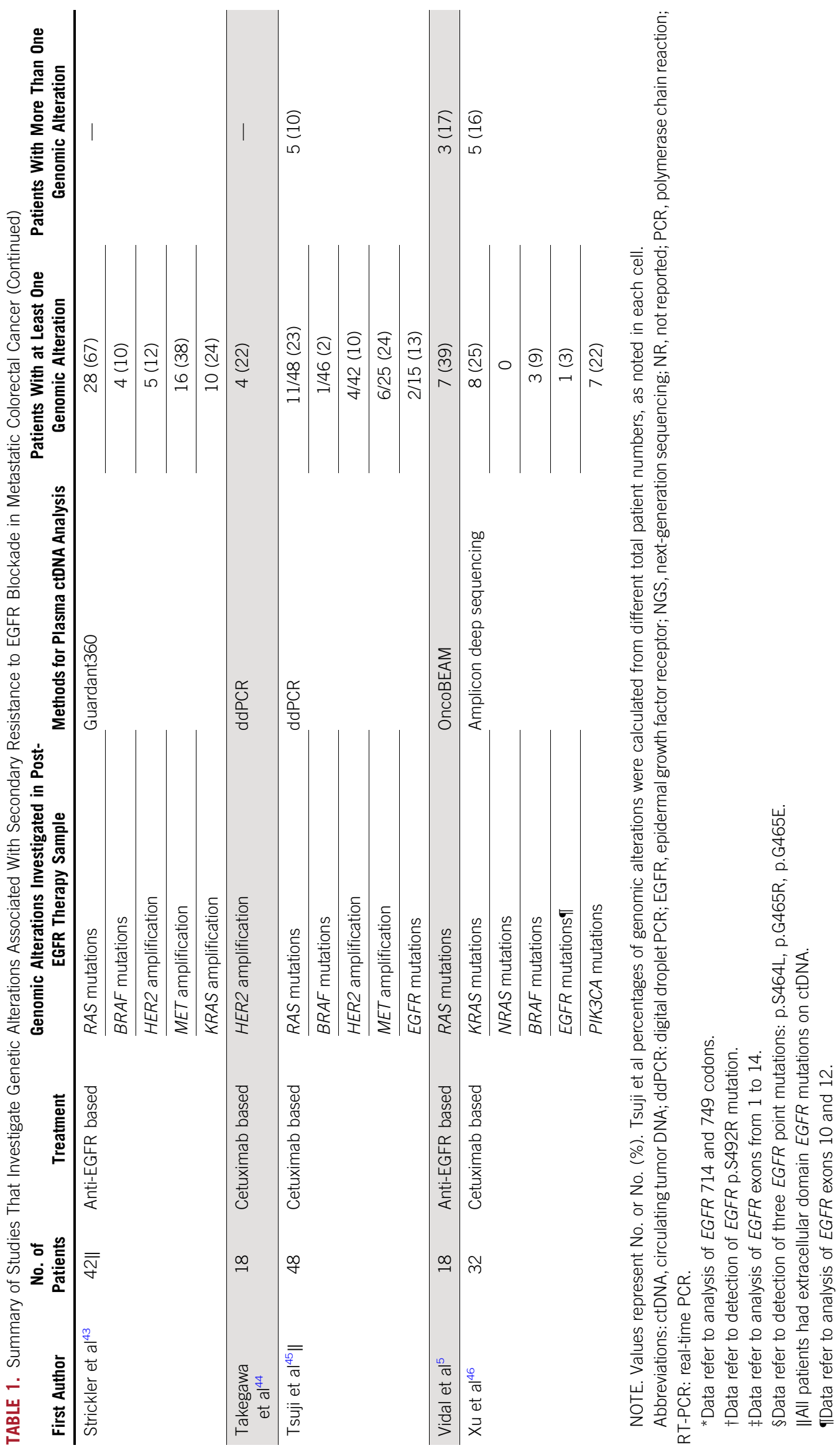




\section{AFFILIATIONS}

${ }^{1}$ Unit of Medical Oncology 2, Azienda Ospedaliera-Universitaria Pisana, Pisa, Italy

${ }^{2}$ Department of Translational Research and New Technologies in Medicine, University of Pisa, Pisa, Italy

${ }^{3}$ Medical Oncology Department, Fondazione IRCCS Istituto Nazionale dei Tumori, Milan, Italy

${ }^{4}$ Department of Oncology and Hemato-oncology, University of Milan, Milan, Italy

Preprint version available on bioRxiv.

\section{CORRESPONDING AUTHOR}

Chiara Cremolini, MD, PhD, Unit of Medical Oncology 2, Azienda Ospedaliera-Universitaria Pisana, and Department of Translational Research and New Technologies in Medicine, University of Pisa, Via Roma, 6756126 Pisa, Italy; e-mail: chiaracremolini@gmail.com.

\section{AUTHOR CONTRIBUTIONS}

Conception and design: Carlotta Antoniotti, Filippo Pietrantonio, Alfredo Falcone, Chiara Cremolini

Collection and assembly of data: Carlotta Antoniotti, Filippo Pietrantonio, Filippo De Braud, Chiara Cremolini

Data analysis and interpretation: Carlotta Antoniotti, Filippo Pietrantonio, Salvatore Corallo, Chiara Cremolini

Manuscript writing: All authors

Final approval of manuscript: All authors

\section{AUTHORS' DISCLOSURES OF POTENTIAL CONFLICTS OF INTEREST}

The following represents disclosure information provided by authors of this manuscript. All relationships are considered compensated.

Relationships are self-held unless noted. I = Immediate Family Member, Inst = My Institution. Relationships may not relate to the subject matter of this manuscript. For more information about ASCO's conflict of interest policy, please refer to www.asco.org/rwc or ascopubs.org/po/author-center.
Filippo Pietrantonio

Consulting or Advisory Role: Amgen, Merck Serono, Bayer, Lilly, Sanofi, Roche, Servier

Salvatore Corallo

Speakers' Bureau: Pierre Fabre

Filippo De Braud

Consulting or Advisory Role: Ignyta, Pfizer, Amgen, Novartis, Daiichi Sankyo, Bristol-Myers Squibb, Servier, Dompè, Pierre Fabre, Roche, Octimet, Incyte

Speakers' Bureau: MSD, Novartis, Bristol-Myers Squibb, Roche, Menarini, Pfizer

Research Funding: Novartis (Inst), Roche (Inst), MSD (Inst), Ignyta (Inst), MedImmune (Inst), Nektar (Inst), Bristol-Myers Squibb (Inst), Merck Serono (Inst), Bayer (Inst), Celgene (Inst), GlaxoSmithKline (Inst), Boehringer Ingelheim (Inst), Lilly (Inst), Pfizer (Inst), Servier (Inst) Travel, Accommodations, Expenses: Roche, Amgen, Bristol-Myers Squibb, Celgene, Daiichi Sankyo

Alfredo Falcone

Honoraria: Lilly, Roche, Merck, Servier, Amgen

Consulting or Advisory Role: Amgen, Bayer, Bristol-Myers Squibb, Lilly, Merck, Roche, Servier

Research Funding: Amgen (Inst), Bayer (Inst), Merck (Inst), Roche (Inst), Sanofi (Inst), MSD (Inst), Servier (Inst)

Travel, Accommodations, Expenses: Amgen, Bayer, Roche, Merck, Servier

Chiara Cremolini

Honoraria: Roche, Amgen, Bayer, Servier

Consulting or Advisory Role: Roche, Bayer, Amgen

Speakers' Bureau: Servier

Research Funding: Merck

Travel, Accommodations, Expenses: Roche, Servier

No other potential conflicts of interest were reported.

\section{REFERENCES}

1. Bettegowda C, Sausen M, Leary RJ, et al: Detection of circulating tumor DNA in early- and late-stage human malignancies. Sci TransI Med 6:224ra24, 2014

2. Denis JA, Guillerm E, Coulet F, et al: The Role of BEAMing and digital PCR for multiplexed analysis in molecular oncology in the era of next-generation sequencing. Mol Diagn Ther 21:587-600, 2017

3. Merker JD, Oxnard GR, Compton C, et al: Circulating tumor DNA analysis in patients with cancer: American Society of Clinical Oncology and College of American Pathologists joint review. J Clin Oncol 36:1631-1641, 2018

4. Baraniskin A, Van Laethem JL, Wyrwicz L, et al: Clinical relevance of molecular diagnostics in gastrointestinal (GI) cancer: European Society of Digestive Oncology (ESDO) expert discussion and recommendations from the 17th European Society for Medical Oncology (ESMO)/World Congress on Gastrointestinal Cancer, Barcelona. Eur J Cancer 86:305-317, 2017

5. Vidal J, Muinelo L, Dalmases A, et al: Plasma ctDNA RAS mutation analysis for the diagnosis and treatment monitoring of metastatic colorectal cancer patients. Ann Oncol 28:1325-1332, 2017

6. Bachet JB, Bouché O, Taieb J, et al: RAS mutation analysis in circulating tumor DNA from patients with metastatic colorectal cancer: The AGEO RASANC prospective multicenter study. Ann Oncol 29:1211-1219, 2018

7. Siravegna G, Mussolin B, Buscarino M, et al: Clonal evolution and resistance to EGFR blockade in the blood of colorectal cancer patients. Nat Med 21:795-801, 2015

8. Schmiegel W, Scott RJ, Dooley S, et al: Blood-based detection of RAS mutations to guide anti-EGFR therapy in colorectal cancer patients: Concordance of results from circulating tumor DNA and tissue-based RAS testing. Mol Oncol 11:208-219, 2017

9. Jones FS, Edelstein D, Wichner K, et al: Performance of standardized BEAMing platform for detecting RAS mutations in the blood of metastatic colorectal cancer (mCRC) patients. J Clin Oncol 34:, 2016 (suppl; abstr 11538)

10. Grasselli J, Elez E, Caratù G, et al: Concordance of blood- and tumor-based detection of RAS mutations to guide anti-EGFR therapy in metastatic colorectal cancer. Ann Oncol 28:1294-1301, 2017

11. Normanno N, Esposito Abate R, Lambiase M, et al: RAS testing of liquid biopsy correlates with the outcome of metastatic colorectal cancer patients treated with first-line FOLFIRI plus cetuximab in the CAPRI-GOIM trial. Ann Oncol 29:112-118, 2018

12. Wan JCM, Massie C, Garcia-Corbacho J, et al: Liquid biopsies come of age: Towards implementation of circulating tumour DNA. Nat Rev Cancer 17:223-238, 2017

13. Ou SI, Nagasaka M, Zhu VW: Liquid biopsy to identify actionable genomic alterations. Am Soc Clin Oncol Educ Book 978-997, 2018

14. Sysmex Inostics: Oncobeam RAS CRC kit. https://www.sysmex-inostics.com/products-services/oncobeam-ras-crc-kit.html 
15. Biocartis: Idylla NRAS-BRAF mutation test.https://www.biocartis.com/meet-idylla/idylla-assays/idylla-nras-braf-mutation-test

16. Biocartis: Idylla KRAS mutation test. https://www.biocartis.com/meet-idylla/idylla-assays/idylla-kras-mutation-test

17. Sepulveda AR, Hamilton SR, Allegra CJ, et al: Molecular biomarkers for the evaluation of colorectal cancer: Guideline from the American Society for Clinical Pathology, College of American Pathologists, Association for Molecular Pathology, and the American Society of Clinical Oncology. J Clin Oncol 35:1453-1486, 2017

18. Van Cutsem E, Cervantes A, Adam R, et al: ESMO consensus guidelines for the management of patients with metastatic colorectal cancer. Ann Oncol 27:1386-1422, 2016

19. National Comprehensive Cancer Network: Clinical Practice Guidelines in Oncology: Colon Cancer, version 4.2018. https://www.nccn.org/professionals/ physician_gls/pdf/colon.pdf

20. Labianca R, Nordlinger B, Beretta GD, et al: Early colon cancer: ESMO Clinical Practice Guidelines for diagnosis, treatment and follow-up. Ann Oncol 24:vi64-vi72, 2013

21. Khan $\mathrm{KH}$, Cunningham $\mathrm{D}$, Werner $\mathrm{B}$, et al: Longitudinal Liquid biopsy and mathematical modeling of clonal evolution forecast time to treatment failure in the PROSPECT-C phase II colorectal cancer clinical trial. Cancer Discov 8:1270-1285, 2018

22. Pietrantonio F, Vernieri C, Siravegna G, et al: Heterogeneity of acquired resistance to anti-EGFR monoclonal antibodies in patients with metastatic colorectal cancer. Clin Cancer Res 23:2414-2422, 2017

23. Ahronian LG, Sennott EM, Van Allen EM, et al: Clinical acquired resistance to RAF inhibitor combinations in BRAF-mutant colorectal cancer through MAPK pathway alterations. Cancer Discov 5:358-367, 2015

24. Corcoran RB, André T, Atreya CE, et al: Combined BRAF, EGFR, and MEK inhibition in patients with $B R A F^{\mathrm{v} 600 \mathrm{E}}$-mutant colorectal cancer. Cancer Discov 8:428-443, 2018

25. Hazar-Rethinam M, Kleyman M, Han GC, et al: Convergent therapeutic strategies to overcome the heterogeneity of acquired resistance in $B R A F^{v 600 E}$ colorectal cancer. Cancer Discov 8:417-427, 2018

26. Oddo D, Sennott EM, Barault L, et al: Molecular landscape of acquired resistance to targeted therapy combinations in BRAF-mutant colorectal cancer. Cancer Res 76:4504-4515, 2016

27. Pietrantonio F, Oddo D, Gloghini A, et al: MET-driven resistance to dual EGFR and BRAF blockade may be overcome by switching from EGFR to MET inhibition in BRAF-mutated colorectal cancer. Cancer Discov 6:963-971, 2016

28. Misale S, Di Nicolantonio F, Sartore-Bianchi A, et al: Resistance to anti-EGFR therapy in colorectal cancer: From heterogeneity to convergent evolution. Cancer Discov 4:1269-1280, 2014

29. Arena S, Bellosillo B, Siravegna G, et al: Emergence of multiple EGFR extracellular mutations during cetuximab treatment in colorectal cancer. Clin Cancer Res 21:2157-2166, 2015

30. Bardelli A, Corso S, Bertotti A, et al: Amplification of the MET receptor drives resistance to anti-EGFR therapies in colorectal cancer. Cancer Discov 3:658-673, 2013

31. Misale S, Yaeger R, Hobor S, et al: Emergence of KRAS mutations and acquired resistance to anti-EGFR therapy in colorectal cancer. Nature 486:532-536, 2012

32. Montagut C, Dalmases A, Bellosillo B, et al: Identification of a mutation in the extracellular domain of the epidermal growth factor receptor conferring cetuximab resistance in colorectal cancer. Nat Med 18:221-223, 2012

33. Yonesaka K, Zejnullahu K, Okamoto I, et al: Activation of ERBB2 signaling causes resistance to the EGFR-directed therapeutic antibody cetuximab. Sci TransI Med 3:99ra86, 2011

34. Russo M, Misale S, Wei G, et al: Acquired resistance to the TRK inhibitor entrectinib in colorectal cancer. Cancer Discov 6:36-44, 2016

35. Diaz LA Jr, Williams RT, Wu J, et al: The molecular evolution of acquired resistance to targeted EGFR blockade in colorectal cancers. Nature 486:537-540, 2012

36. Misale S, Arena S, Lamba S, et al: Blockade of EGFR and MEK intercepatients heterogeneous mechanisms of acquired resistance to anti-EGFR therapies in colorectal cancer. Sci Transl Med 6:224ra26, 2014

37. Mohan S, Heitzer E, Ulz P, et al: Changes in colorectal carcinoma genomes under anti-EGFR therapy identified by whole-genome plasma DNA sequencing. PLoS Genet 10:e1004271, 2014

38. Morelli MP, Overman MJ, Dasari A, et al: Characterizing the patterns of clonal selection in circulating tumor DNA from patients with colorectal cancer refractory to anti-EGFR treatment. Ann Oncol 26:731-736, 2015

39. Newhall K, Price T, Peeters M, et al: Frequency of S492R mutations in the epidermal growth factor receptor: Analysis of plasma DNA from metastatic colorectal cancer patients treated with panitumumab or cetuximab monotherapy. Ann Oncol 25:ii109, 2014 (suppl; abstr 0-0011)

40. Raghav K, Morris V, Tang C, et al: MET amplification in metastatic colorectal cancer: An acquired response to EGFR inhibition, not a de novo phenomenon. Oncotarget 7:54627-54631, 2016

41. Price TJ, Peeters M, Boedigheimer M, et al: Clinical outcomes and emergent circulating tumor (ct)DNA RAS mutations and allele fraction for patients with metastatic colorectal cancer (mCRC) treated with panitumumab from the ASPECCT study. J Clin Oncol 35, 2017 (suppl; abstr 3584)

42. Siena S, Sartore-Bianchi A, Garcia-Carbonero R, et al: Dynamic molecular analysis and clinical correlates of tumor evolution within a phase II trial of panitumumab-based therapy in metastatic colorectal cancer. Ann Oncol 29:119-126, 2018

43. Strickler JH, Loree JM, Ahronian LG, et al: Genomic landscape of cell-free DNA in patients with colorectal cancer. Cancer Discov 8:164-173, 2018

44. Takegawa N, Yonesaka K, Sakai K, et al: HER2 genomic amplification in circulating tumor DNA from patients with cetuximab-resistant colorectal cancer. Oncotarget 7:3453-3460, 2016

45. Tsuji Y, Shitara K, Yamanaka T, et al: REVERCE: Randomized phase II study of regorafenib followed by cetuximab versus the reverse sequence for metastatic colorectal cancer patients previously treated with fluoropyrimidine, oxaliplatin, and irinotecan-Biomarker analysis. J Clin Oncol 36, 2018 (suppl; abstr 3510)

46. Xu JM, Wang Y, Wang YL, et al: PIK3CA mutations contribute to acquired cetuximab resistance in patients with metastatic colorectal cancer. Clin Cancer Res 23:4602-4616, 2017

47. Montagut C, Argilés G, Ciardiello F, et al: Efficacy of Sym004 in patients with metastatic colorectal cancer with acquired resistance to anti-EGFR therapy and molecularly selected by circulating tumor DNA analyses: A phase 2 randomized clinical trial. JAMA Oncol 4:e175245, 2018

48. Santini D, Vincenzi B, Addeo R, et al: Cetuximab rechallenge in metastatic colorectal cancer patients: How to come away from acquired resistance? Ann Oncol 23:2313-2318, 2012

49. Parseghian CM, Loree JM, Morris VK, et al: Anti-EGFR resistant clones decay exponentially after progression: Implications for anti-EGFR re-challenge. Ann Oncol doi: 10.1093/annonc/mdy509 [Epub ahead of print on November 21, 2018] 
50. Cremolini $\mathrm{C}$, Rossini $\mathrm{D}$, Dell'Aquila $\mathrm{E}$, et al: Rechallenge for patients with $R A S$ and $B R A F$ wild-type metastatic colorectal cancer with acquired resistance to firstline cetuximab and irinotecan: A phase 2 single-arm clinical trial. JAMA Oncol, 2018

51. ClinicalTrials.Gov: CHRONOS trial. https://clinicaltrials.gov/ct2/show/NCT03227926 


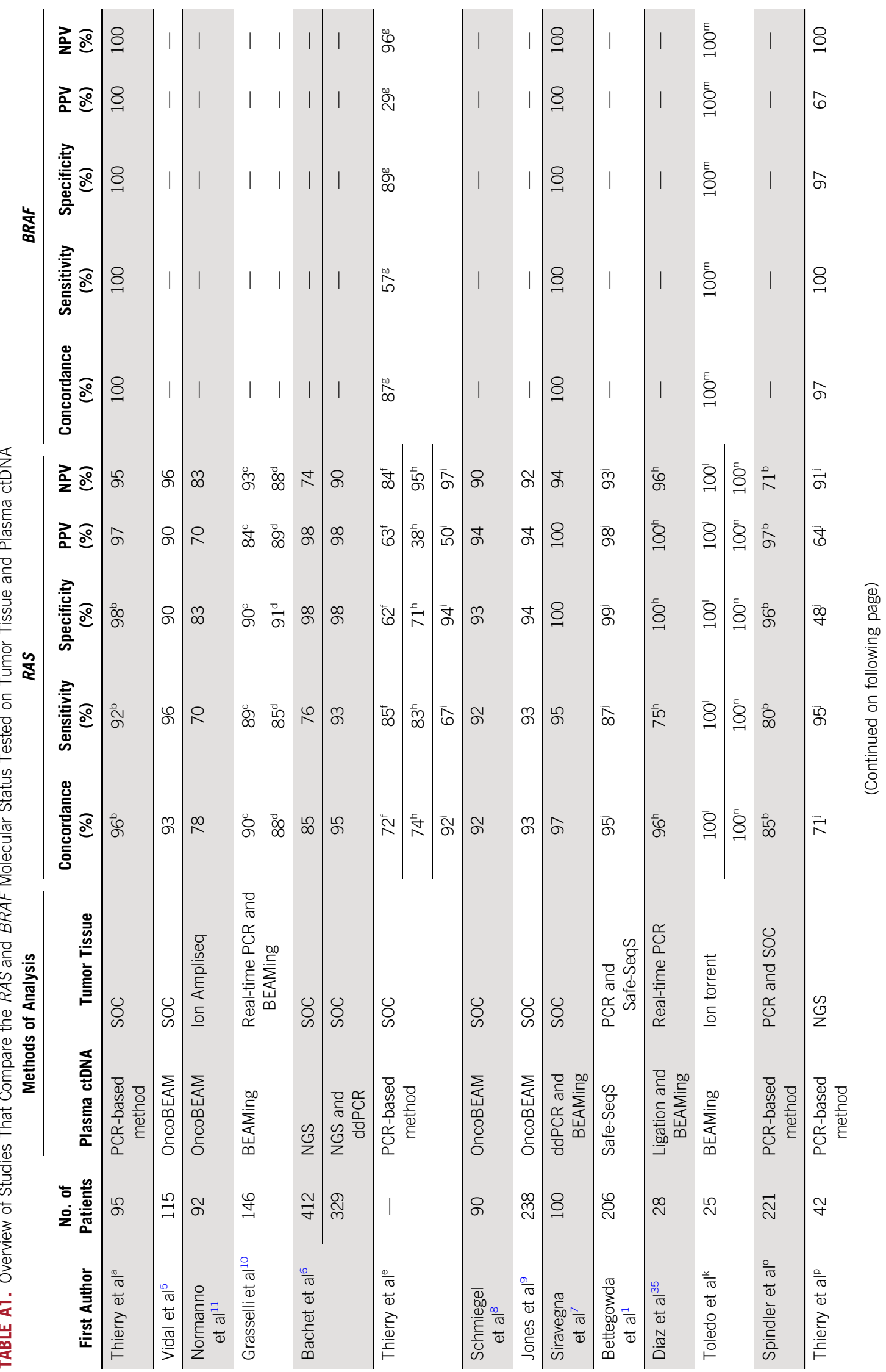




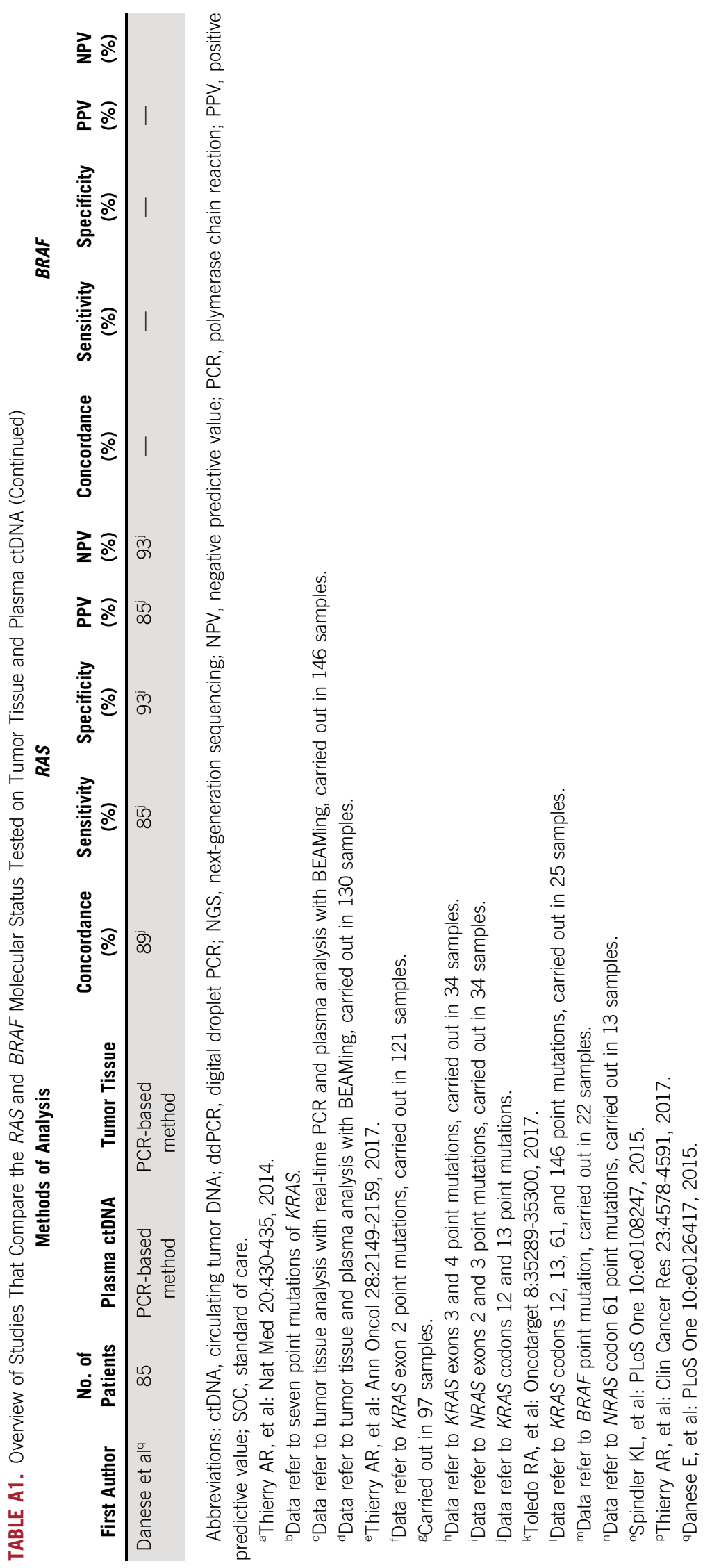

10 @ 2019 by American Society of Clinical Oncology 


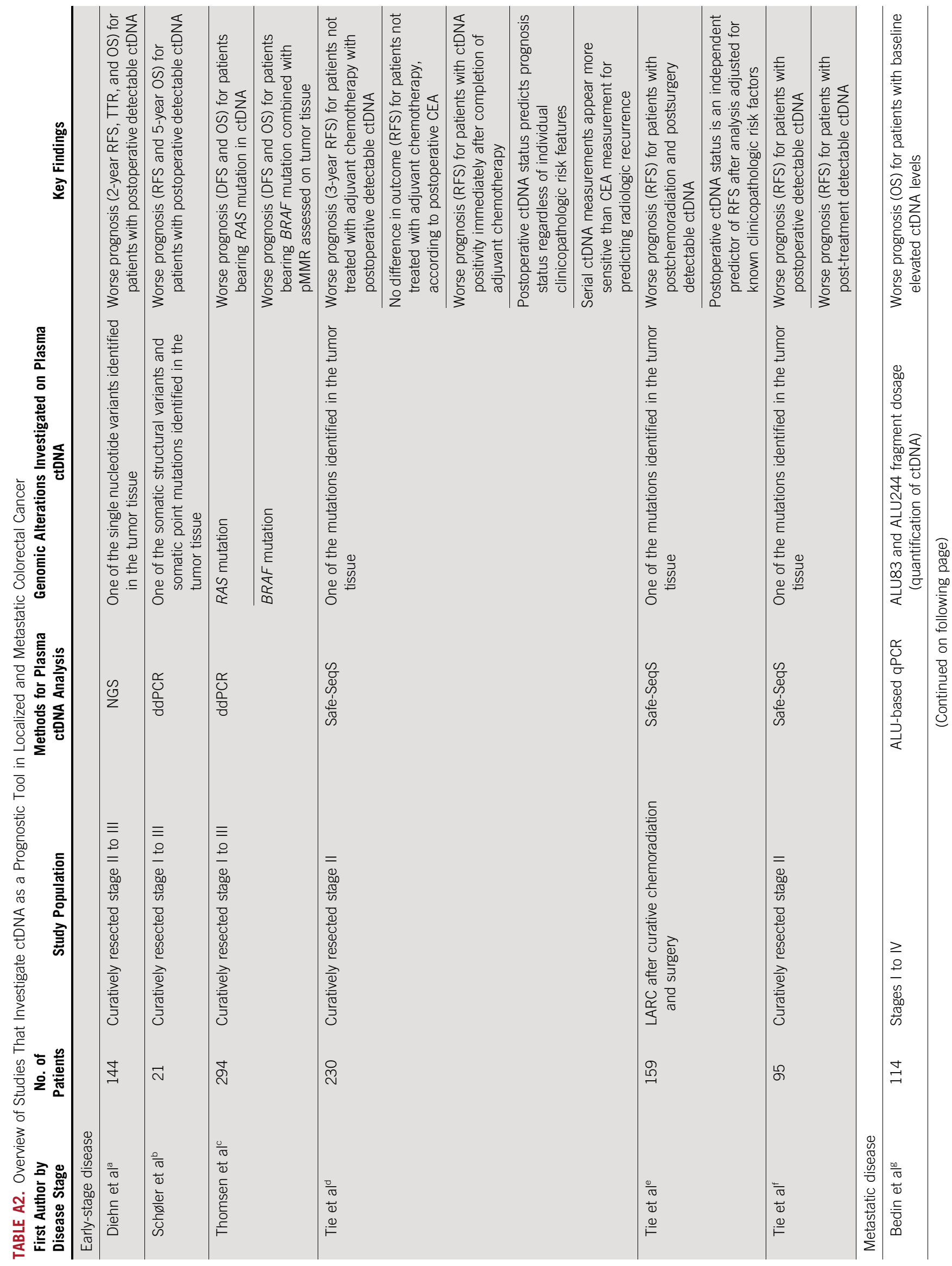




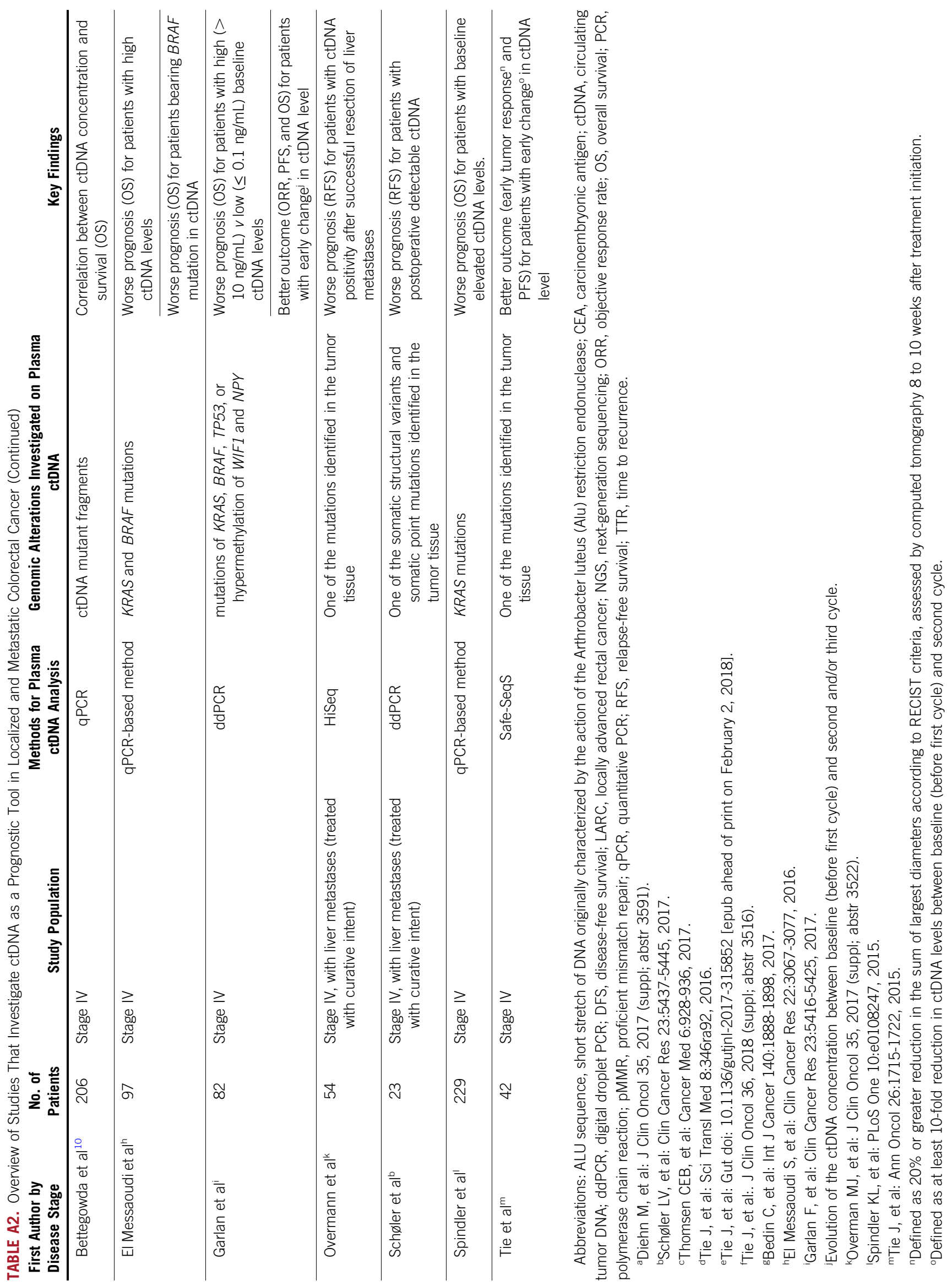

12 (C) 2019 by American Society of Clinical Oncology 


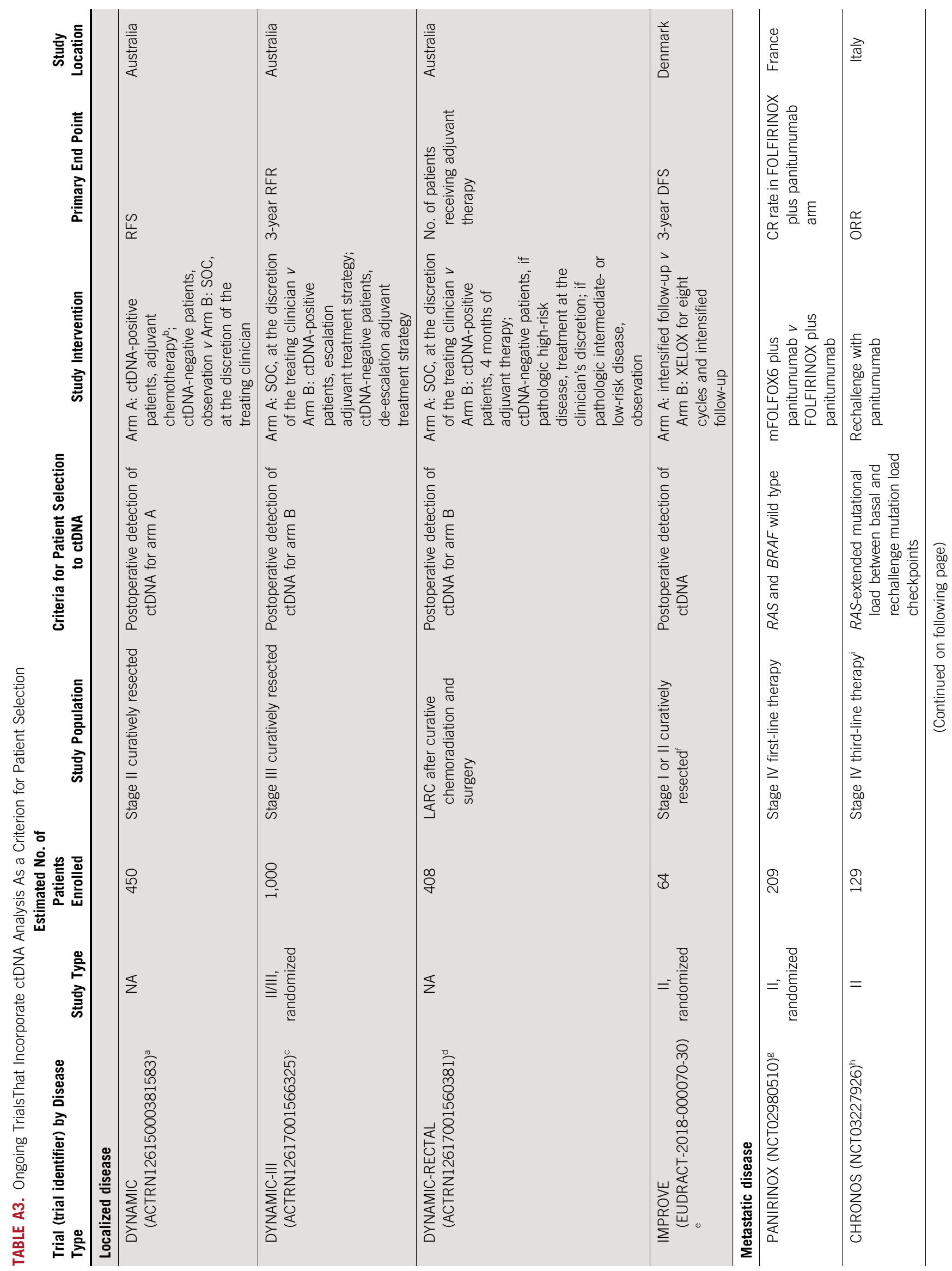




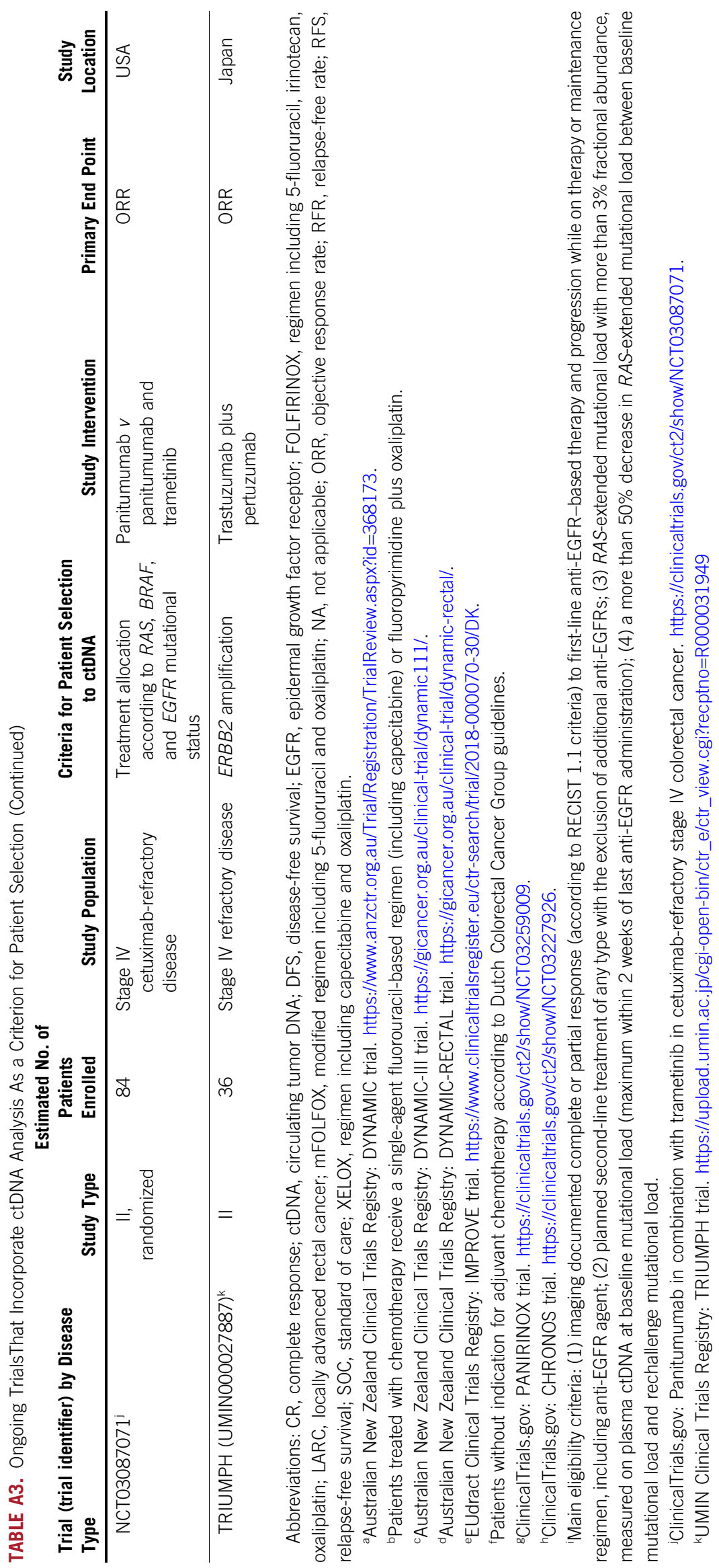

14 ㅇ 2019 by American Society of Clinical Oncology 